\title{
Reactive power optimization of power system based on niching differential evolution algorithm
}

\author{
ZHANG Xiao Fei ${ }^{1, \mathrm{a}}$, GUO Xiang Fu ${ }^{1, \mathrm{~b}}$, YUAN Li Hua ${ }^{2, \mathrm{c}}$ \\ ${ }^{1}$ No.85 Songshan south Road, Henan province zhengzhou, China \\ ${ }^{2}$ No.1200 Cangyuan Road, Minhang District Shanghai, China \\ a zhangxiaofei@ha.sgcc.com.cn, ${ }^{b}$ guoxiangfu@ha.sgcc.com.cn, ${ }^{c} y u a n l i h u a$ \\ 911@163.com
}

\begin{abstract}
Reactive power optimization is the non-linear integer problems, suitable reactive power compensation can reduce the transmission losses and guarantee the power system operate stability, safety and economy. The differential evolution algorithm is the intelligent evolutionary algorithm had fast and precision convergence. In this paper, improved differential evolution algorithm aiming at the model of reactive power optimization, and introduces the niche theory. Then to enhance the local search ability of algorithm and broaden the search range, the speed and precision of convergence has different degrees of improvement. At last, the paper selects several algorithms that acquire excellent results on reactive power optimization, and then compares algorithms to confirm advantage of improving the differential evolution algorithm.
\end{abstract}

Key words : Reactive power optimization; differential evolution algorithm; niche; particle swarm optimization.

\section{Introduction}

Reactive power optimization problem of system is a mixed nonlinear programming problem with multivariable and multi-constrained. Its controlled variable both has continuous variables and discrete variables. The optimization process is very complicated, with the large-scale computing. From the traditional algorithm (such as linear programming and nonlinear programming) to the artificial intelligence algorithms (such as particle swarm optimization algorithm and genetic algorithm) make a contribution to reactive power optimization in different degree ${ }^{[1]}$.

With the development of intelligent heuristic optimization algorithm, differential evolution algorithm has been gradually applied to power system. The algorithm with the characteristics of easy understanding and parallel processing and good robustness, could high probability find the global optimal solution of problem. And its computational efficiency is higher than traditional evolutionary programming algorithm. The biggest advantage is simple, easy to implement, 
fast convergence speed, and high accuracy search. It is not only suitable for scientific research, but also suitable for engineering application. So when differential evolution algorithm is been put forward, immediately aroused the attention of researchers in the fields of evolutionary computation and published stacks of studies. It has been successfully applied in field of function optimization, neural network design, classification, pattern recognition, signal processing, robot technology etc ${ }^{[2]}$.

Differential evolution algorithm achieves the optimization objective by simulating natural phenomena and the swarm behavior of biological swarm in nature. The algorithm has better optimization efficiency, no strict feasibility requirement for initial solution, no manual intervention, and has no analytical requirements for constraint function and objective function. There are three mainly operations: mutation, crossover and selection. It is easily implement, simple operation and local search ability. So it is Suitable for reactive power optimization of power system.

\section{Reactive power optimization model}

The goal reactive power optimization is that in the guarantee of reactive power balance the system network meets security constraints and minimizes active power loss, by adjusting the voltage of generator, transformer ratio and shunt capacitor. The mathematical model can be expressed as follow ${ }^{[3]}$.

$$
\min F=\sum_{\substack{i \in N \\ j \in I}} G_{i j}\left(U_{i}^{2}+U_{j}^{2}-2 U_{i} U_{j} \cos \theta_{i j}\right)+\lambda_{1} \sum_{\alpha}\left(\frac{U_{i}-U_{i l \lim }}{U_{i \max }-U_{i \min }}\right)^{2}+\lambda_{2} \sum_{\beta}\left(\frac{Q_{i}-Q_{i l \mathrm{im}}}{Q_{i \max }-Q_{i \min }}\right)^{2}
$$

Among them, $\mathrm{F}$ is the target function; $\lambda_{1}$ and $\lambda_{2}$ are respectively the penalty factors in violation of voltage constraints and reactive power generation constraints; $\alpha$ and $\beta$ are the node set in violation of node voltage constraints and reactive power generation constraints; $U_{i}, U_{\text {imax }}$ and $U_{\text {imin }}$ are the node voltage and upper and lower limit of node voltage; $U_{\text {ilim }}$ and $Q_{i l i m}$ are voltage and reactive power limit of the node $i$. The formula is defined as follows ${ }^{[4]}$.

$$
\begin{array}{r}
U_{i \lim }= \begin{cases}U_{i \max }, & U_{i}>U_{i \max } \\
U_{i \min }, & U_{i}<U_{i \min }\end{cases} \\
Q_{i \lim }= \begin{cases}Q_{i \max }, & Q_{i}>Q_{i \max } \\
Q_{i \min }, & Q_{i}<Q_{i \min }\end{cases}
\end{array}
$$

Equality constraints are written as follows. 


$$
\left\{\begin{array}{l}
P_{i}-U_{i} \sum_{j=1}^{N} U_{j}\left(G_{i j} \cos \theta_{i j}+B_{i j} \sin \theta_{i j}\right)=0 \\
Q_{i}-U_{i} \sum_{j=1}^{N} U_{j}\left(G_{i j} \sin \theta_{i j}-B_{i j} \cos \theta_{i j}\right)=0
\end{array}\right.
$$

Inequality constraints of state variable are written as follows.

$$
\left\{\begin{array}{l}
U_{G \text { min }} \leq U_{G} \leq U_{G \text { max }} \\
K_{T \text { min }} \leq K_{T} \leq K_{T \text { max }} \\
Q_{c \text { min }} \leq Q_{c} \leq Q_{c \text { max }} \\
U_{L \text { min }} \leq U_{L} \leq U_{L \text { max }} \\
Q_{G \text { min }} \leq Q_{G} \leq Q_{G \text { max }}
\end{array}\right.
$$

In the formula, $U_{G \min }$ and $U_{G \max }$ are upper and lower limit value of the generator terminal voltage; $K_{T \min }$ and $K_{\text {Tmax }}$ are upper and lower limit value of adjustable transformer tap changer; $Q_{C \min }$ and $Q_{C \max }$ are upper and lower limit value of the compensation capacitor switched groups; $U_{L \min }$ and $U_{L \max }$ are upper and lower limit value of load points' voltage; $Q_{G \min }$ and $Q_{G \max }$ are upper and lower limit value of reactive power generation ${ }^{[5]}$.

\section{Application of improved differential evolution algorithm in reactive power optimization}

\subsection{Improved differential evolution algorithm}

Improvement of the basic differential evolution algorithm mainly has the following aspects ${ }^{[6-9]}$.

A. The choice difference individual

This paper will add Fitness Eulidean-distance Ration (FER) to differential evolution algorithm increasing the diversity of swarm. Fitness Eulidean-distance ration was first introduced to particle swarm optimization algorithm (FERPSO) for solving multimodal optimization problems. Joining this mechanism makes the particles move in near and best from it. In the FERPSO, the near best one of each individual is largest FER one of neighborhood individual. The calculating formula of FER as follows.

$$
\begin{aligned}
& \operatorname{FER}_{(j, i)}=\alpha \cdot \frac{f\left(p_{j}\right)-f\left(p_{i}\right)}{\left\|p_{j}-p_{i}\right\|} \\
& \alpha=\frac{\|s\|}{f\left(p_{g}\right)-f\left(p_{w}\right)}
\end{aligned}
$$

Among them, $p_{i}$ and $p_{j}$ respectively represent optimal individual of the $i$ and the $j$ individual. $\alpha$ is a proportional coefficient; $p_{w}$ is worst-fitness individual in 
current swarm; $\|S\|$ is the size of the search space.

$$
\|S\|=\sqrt{\sum_{k=1}^{d}\left(x_{k}^{u}-x_{k}^{l}\right)^{2}}
$$

In formula, $x_{k}^{u}$ and $x_{k}^{l}$ is the maximum and minimum values of the $\mathrm{K}$ dimensions search space.

FER method is applied to differential evolution algorithm. Through the method to choice $x_{r 1}$ to $x_{r 5}$ five individuals, and the calculating formula of FER is written as follows.

$$
\mathrm{FER}_{(j, i)}=\frac{\operatorname{normalize}\left(f\left(p_{j}\right)-f\left(p_{i}\right)\right)}{\text { normalize }^{N}\left(\left\|p_{j}-p_{i}\right\|\right)}
$$

Considering that $\alpha$ is a constant, if delete the proportion coefficient, it will have no effect on sequence of FER, meanwhile reduce the computational complexity and improve the efficiency of the algorithm. FER's denominator is normalized. At same time, in order to strengthen distance's function to keep diversity of swarm, denominator is become the n-th power of distance. The value of $\mathrm{N}$ adjusts according to search condition. According to the above formula, calculate every individual to all swarms' individuals of FER, and put them in order. And then according the roulette wheel method select $x_{r 1}$ to $x_{r 5}$ individuals in other individuals.

In the course of swarm search, there may be many individuals gathered at a peak. It will cause the waste of search, so initialize a part of worst fitness individuals for remaining elite parts continue to search the peak. The remained elite individuals don't use FER formula any longer, but use standard differential evolution algorithm to local search ensuring the convergent precision of algorithm. In order to ensure that the swarm can continue to use FER strategy, add the individuals had same number of elite individuals to initialized swarms. It can global search optimal solution. So the size of swarm NP is adaptively adjusted.

B. Memory mechanism added in, algorithm will save optimal solution's position what elite individuals has searched every time. Because dynamic problems is a periodic function, it is necessary that memory mechanism join in algorithm. At same time, it can use the saved information in the process of swarm's initialization.

C. The variation factor $\mathrm{F}$ is decreasing after swarm's initialization and then lineal increasing in the search process. It's helpful to splitting swarm.

\subsection{Differential evolution algorithm based on Fitness Eulidean-distance Ration}

The procedure of improved different evolution algorithm as follows. 


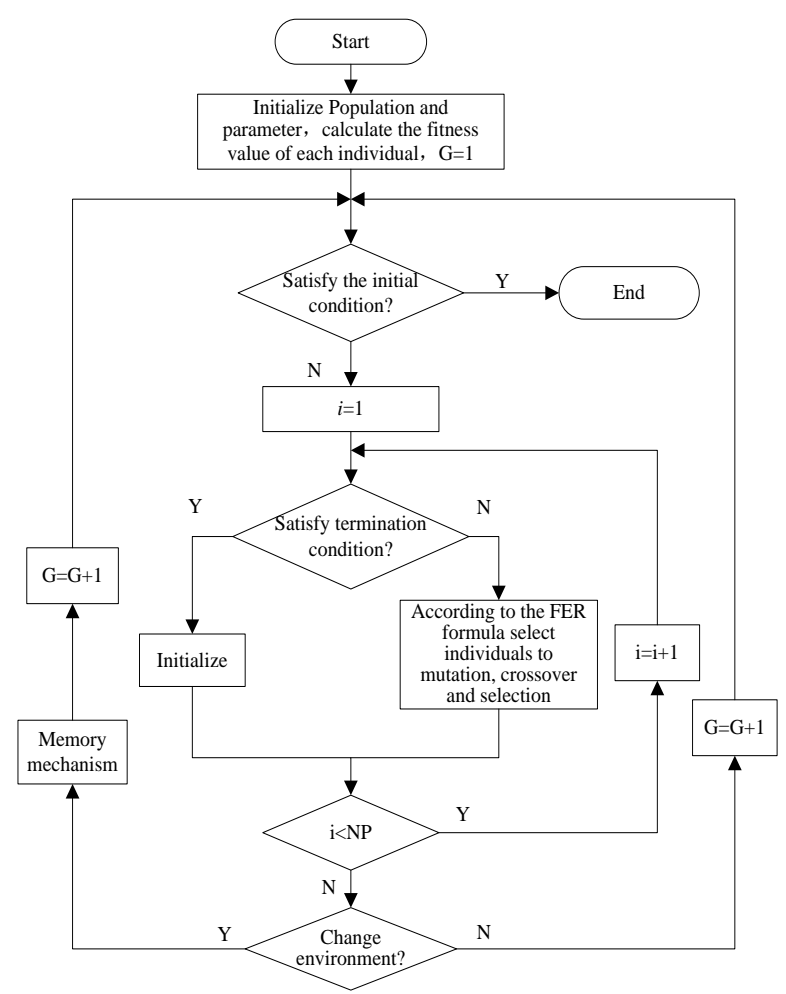

Fig.1 The procedure of FER different evolution algorithm

Improved differential evolution algorithm based on FER is applied in the reactive power optimization of power system. Search range and precision are improved on the original algorithm. Firstly, the paper establish power system reactive power optimization model, and then compare application of reactive power optimization with the particle swarm optimization algorithm, differential evolution algorithm and niching particle swarm algorithm. To show the superiority of improved strategy .

\section{Application procedure}

A. Initialize algorithm parameters and system parameter

At first, initialize swarm size $M$, max iteration and cross rate. And then initialize output power of PV point, system load and initialize and phase angle of system nodes.

B. Power flow calculation According to the Newton-Raphson method

Set the maximum iteration in the biggest received error and the error of the Newton-Raphson method. Then use Newton-Raphson method to power flow calculation.

C. Variation

Through the calculation of FER select individual of traction variation, in the meantime, linear adjust the mutation factor F. At time of extending the search 
scope, increase the search precision and convergence speed. After the FER search process, it will form a number of small swarms, in the swarm. Deepen the local search in the guidance of locally optimal particle.

D. Crossover and selection

After Variation, it is the vector $V_{i}^{k+1}$. Interlaced operate it and the original vector to obtain the test vector. Then select the better fitness between the original vector and test vector, and keep it in a swarm.

E. Verification of termination criterion

If the present iteration $\mathrm{k}$ is not equal to the maximum iteration, then turn to step $\mathrm{B}$ and recalculate. If the present iteration $\mathrm{k}$ is equal to the maximum iteration, then terminate and Write the power system loss after optimization. It can also set a convergence precision as the condition of iteration stop, stopping search when it reaches a precision. The combination of the two can save search time wonderfully.

\section{The example analysis}

In order to verify the advantage of differential evolution algorithm based on Fitness Eulidean-distance Ration, this paper solve optimization problems in power system respectively using particle swarm optimization, differential evolution, niche particle swarm optimization and Fitness Eulidean-distance Ration differential evolution. (The results see Tab 1)

Tab.1 Comparison of different optimization results

\begin{tabular}{|c|c|c|c|c|c|}
\hline $\begin{array}{c}\text { Test } \\
\text { system }\end{array}$ & Algorithm & $\begin{array}{c}\text { Optimal } \\
\text { network } \\
\text { loss }\end{array}$ & $\begin{array}{c}\text { Worst } \\
\text { network } \\
\text { loss }\end{array}$ & $\begin{array}{c}\text { Average } \\
\text { network loss }\end{array}$ & $\begin{array}{c}\text { standard } \\
\text { deviation }\end{array}$ \\
\hline \multirow{4}{*}{ IEEE57 } & PSO & 0.0648 & 0.0695 & 0.0662 & 0.0089 \\
\cline { 2 - 6 } & DE & 0.0645 & 0.0668 & 0.0656 & 0.0073 \\
\cline { 2 - 6 } & NPSO & 0.0640 & 0.0642 & 0.0640 & 0.00586 \\
\cline { 2 - 6 } IEEE30 & FERDE & 0.00634 & 0.00782 & 0.00692 & $7.32 \mathrm{e}-006$ \\
\cline { 2 - 6 } & PSO & 0.06064 & 0.06265 & 0.06471 & 0.0009 \\
\cline { 2 - 6 } & DE & 0.06042 & 0.06138 & 0.06345 & 0.0003 \\
\cline { 2 - 6 } & FPSO & 0.05963 & 0.06081 & 0.06000 & 0.0001 \\
\hline & PSDE & 0.00517 & 0.00611 & 0.00581 & $2.48 \mathrm{e}-007$ \\
\cline { 2 - 6 } IEEE14 & 0.05392 & 0.06142 & 0.06001 & 0.00004 \\
\cline { 2 - 6 } & DE & 0.05134 & 0.05724 & 0.05532 & 0.00007 \\
\cline { 2 - 6 } & FPSO & 0.04927 & 0.05421 & 0.05271 & 0.00003 \\
\hline
\end{tabular}

By comparing the worst network loss, it reached that FERDE got the minimum network loss in the four algorithms through calculation of three systems: IEEE 14, IEEE 30 and IEEE 57. And the worst loss and average loss of FERDE algorithm in independent experiment is least among the four algorithms.

In the stability optimization, for the reactive power optimization of three systems IEEE 14, IEEE 30 and IEEE 57, FERDE algorithm can obtain the 
minimum standard deviation of network loss and optimized stability of best algorithm.

To sum up, when the FERDE separately solve reactive power optimization of three systems, obtain a better optimal performance than the DE, PSO, and NPSO algorithm. And result shows the FERDE is successfully applied in search process of reactive power optimization.

\section{Conclusion}

Reactive power optimization can improve the security of electric power system and effectively reduce the network loss of power system. This paper studies differential evolution algorithm based on Fitness Eulidean-distance Ration and improves basic differential evolution algorithm. It improves the convergence speed and solution's quality of basic differential evolution algorithm. So it is a good algorithm to solve the problem of reactive power optimization, reducing power system network loss in the precondition of security and reliability of the power systems. Therefore it is well worth researchers delving.

\section{References}

[1] Holland J H. Adaptation in natural and artificial systems[M]. University of Michigan Press, Ann Arbor, MI, 1975.

[2] Jin li-zhong, Chang Gui-ran, Jia Jie, Node distribution optimization in mobile sensor networks based on differential evolution algorithm[J], Control and Decision, 2010, 25(12): 1857-1860.

[3] Li Xin-bin, Zhu Qing-jun, Ma Hong-xia, Overview of particle swarm optimization algorithm on reactive optimization for power system, Journal of Yanshan University, 2008, 32(3): 13-16.

[4] Tang Li-feng, Wei Zhi-nong, Huang Ting, Zhang Jian-ping, Improved differential evolution algorithm for fault location of distribution networks[J], Proceedings of the CSU-EPSA, 2011, 23(1): 17-21.

[5] Xu Wen-chao, Guo Wei, Sunnarize of reactive power optimization model and algorithm in electric power system[J], Proceedings of the CSU-EPSA, 2003, 15(1):100-105.

[6] Tan Tao-liang, Zhang YAO, Reactive power optimization based on genetic/tabu search hybrid algorithm [J], Power System Technology, 2003,28(11).

[7] Qin A K, Huang V L, Suganthan P N. Differential evolution algorithm with strategy adaptation for global numerical optimization [J]. Evolutionary Computation, IEEE Transactions on, 2009, 13(2): 398-417.

[8] Yang Z, Tang K, Yao X. Self-adaptive differential evolution with neighborhood search[C]. Evolutionary Computation, 2008. CEC 
2008.(IEEE World Congress on Computational Intelligence). IEEE Congress on. IEEE, 2008: 1110-1116.

[9] Qin A K, Suganthan P N. Self-adaptive differential evolution algorithm for numerical optimization[C]. Evolutionary Computation, 2005. The 2005 IEEE Congress on. IEEE, 2005, 2: 1785-1791. 